\title{
Distributed Teaching Model based on Flipped Classroom for HF Electronic Circuit
}

\author{
Danyang Qin*, Hong Wu, Erfu Wang, Hongbin Ma, Qun Ding \\ Electronic Engineering College, Heilongjiang University \\ Harbin, Heilongjiang 150080, P.R.China \\ *qindanyang@hlju.edu.cn
}

\begin{abstract}
Rapid development of modern communication has a close relationship with the support of information talents, who benefit from the continuous perfection of discipline establishment. Partial content in HF Electronic Circuit is taken as the reform object, and the flipped classroom is taken as the reform means. Study and analysis are performed from teacher-student role exchange, preclass teaching resource perfection, in-class teaching means improvement and post-class evaluation adjustment, so as to take full advantage of flipped classroom and realize hierarchical and asynchronous training in real significance. The proposed completed teaching system will make great contribution to talent training quality for electronic information and teaching research level of the whole discipline.
\end{abstract}

Keywords-flipped classroom; distributed teaching; HF electronic circuit; situation creation.

\section{INTRODUCTION}

Higher education is an important way to modern cultural thoughts and technology propagation, carrying the sacred mission to enhance the national quality and national scientific strength[1,2]. Classroom teaching is the key and core to the education, the method and effect of which will influence the talents training quality directly. Development of computer, multimedia and internet technology fast promotes the informatization. While the internet and interaction covers every corner of the earth, teaching method, teaching targets, teaching models, teaching processes and teaching roles will change greatly. The "Education Informatization Ten Years Development Plan (2011-2020)" indicated that the development of educational informatization should take the education concept innovation as the kernel, take high quality education resource and learning environment as the basis, and take learning style and educational pattern innovation as the key.

Institution of higher learning is the cradle for high-quality talents training, and the creative teaching pattern development has a great relationship with the creative ability training. Dr. Zhong Qiquan in East China Normal University emphasized that the intercommunication by life experience and media between teachers-students and students- students would be the only means to make the knowledge be delivery[3]. It shows that the dialogue and communication are the main way for higher education. The knowledge delivery, however, will not be restricted in classroom. With the rising international competitive and the transformation of the quantitative change to qualitative change for higher education, the improvement of educational effect and efficiency becomes the important target in higher education and teaching reform, which takes the informatization and initiative as the entry point. The former is the indication from teaching method, mainly referring to rapid technology alternation, knowledge propagation in time and ubiquitous teaching objects; the latter is the indication from properties of teaching objects, mainly referring to the positive student attitude, reasonable organization and various materials.

\section{TRanditional Teaching Method Analysis For HF Electronic CiRCUIT}

Rapid development of modern communication has a close relationship with the support of information talents, who benefit from the continuous perfection of discipline establishment. For the information and communication discipline in China, the Electronic Engineering discipline in Euramerica, as well as the information and intelligence discipline in Korea and Japan, HF Electronic Circuit is an important professional basic course, by which students will understand the basic components and working principle of transmitting and receiving circuits, and grasp the analytical method and practical application of HF system, so as to lay the foundation for later theoretical study and experiment. High requirement of theoretical property, engineering character and practicalness, combing with abstract and complicated circuits, make HF Electronic Circuit one of the most difficult professional courses. Therefore, colleges and universities keep trying to adopt new teaching pattern and perform relative reform. Blackboardwriting method with low efficiency is replaced by plenty of multimedia, network and practical teaching. These reforms, naturally, are aiming at the forms but not the essence. For example, PPT adopted in multimedia is electronic blackboard-writing; on-line education is either video film of classroom teaching or the PPT with voice. Nearly all patterns take the basis of traditional paradigm 
by concept and definition-principle and method-calculation and derivation-formula and conclusion. Above all, main problems in HF Electronic Circuit traditional teaching method are as follows:

\section{A. Difficulty in courseiItself will reduce the learning confidence and interest.}

HF Electronic Circuit takes Circuit Analysis, Analog Electrical Circuitry, Digit Circuit, etc. as the basis. The fact that many concepts, equations, circuits and analysis methods in such course gives rise to the lack of learning interest, and even cause the fear of difficulty, which will bring out the shortage of learning motive and low confidence.

\section{B. Limited period will cause learning bottleneck easily.}

All professional courses have the limited period. The efficiency of intensive classes seems high. That, however, is not the case, since the students cannot keep the concentration for long. Survey shows that, according to the case with 50min per teaching period, $44 \%$ of the students indicate the information will be saturated in $20 \mathrm{~min}$ to $30 \mathrm{~min} ; 17 \%$ indicate the thinking efficiency will decline with 20min continuously learning. Though Class Fragmentation could increase the class efficiency and prolong the information saturated time to some degree, it is not satisfactory in the view of increasing the amount of information capacity. Actually, the contradiction between the limited learning period and the declining absorption curve cause the drop of the classroom efficiency.

\section{Full knowledge will be hardly captured by same-course-same-paper.}

In professional courses, knowledge points can be obtained by combing with the practical examples or application cases. The application problems, however, always involve lots of parameters and factors. Such problems themselves have practical significance indeed, but the lack or disconnection of any knowledge points will cause the test fail. The purpose of the exam never lies in the scores, but the knowledge delivery itself. There exists difference in intelligence and capability for different students. Stratified teaching cannot be satisfied by same-course-same-teaching and same-teaching-same-period for traditional teaching.

\section{Distributed TeACHING PATtern BASED ON FLIPPED ClassRoOM}

After Salman Khan shared the math teaching video experience in Khan Academy on TED annual meeting in 2011, a new classroom pattern called flipped classroom came into the sight of educationalist. With open courseware in MIT, open class in Yale and Micro-lecture in Khan Academy springing up, flipped classroom begin to appear in colleges and universities[4]. Up to 2016 end, there are 65cities in 9 countries carrying out the exploration and teaching reform about flipped classroom. In higher education field of China, the introduction of flipped classroom always appears in disciplines like literature, history, philosophy and languages rather than science and engineering area, which is because of the subject feature[5]. In existing exploration, simple flipped form is easy to perform, but the real flipped effect is hard to obtain. Therefore, the form of flipped classroom is not the key of education, but how to use it to improve the effect and efficiency is. Taking creating e-learning environment and boosting innovation about learning style and teaching pattern as the basic principle, partial content in HF Electronic Circuit as the key reform goal, and flipped classroom as the reform measure, this paper will research and analyze through teachers-students role exchange, pre-class materials perfection, in-class teaching method innovation and post-class evaluation adjustment, in order to take full advantages of flipped classroom and break the traditional patter of same-course-same-paper. Distributed teaching method is proposed to realize hierarchical and classified teaching in true sense. Combining with the e-learning environment and the superiority of traditional teaching style, a complete theory teaching system is established, including learning mechanism, teaching pattern, course content and performance appraisal method, which has a net structure with two vertical dimensions as contend-oriented dimensionality and course-oriented dimensionality. Content-oriented dimensionality mainly considers the content modular, including:

(1) Active Learning Mechanism Establishment. Students' centrality and teachers' leading character will be focused on with flipped classroom based distributed teaching mechanism on HF Electronic Circuit. During the whole process, guidance and enlightenment should be brought to higher position by weakening the monologue of the teacher as well as strengthening the subject consciousness of the students. The important means to ensure the flipped pattern is the Illuminating problems, which will lead the students to finish the pre-class learning. For example, In Chapter 1 about the communication system there may be the questions: Why does the wireless message need to be modulated, and could massage be passing without modulation? In Chapter 3 about HF power amplifier there may be the questions: Can we obtain the maximum power and the efficiency at the same time? In post-class part, students will be guided to summarize and review so as to change from the passive receivers to positive participants.

(2) Distributed Teaching Paradigm Construction. Synchronization is a classical feature in traditional teaching with taking all the students as a whole. Considering the difference in the ability of understanding and knowledge assimilation, the distributed teaching mechanism based on flipped classroom proposed in this paper will change concentration to distribution, break whole class into models and take on-demand instead of on-period. Moreover, the establishment of personalized learning environment will realize differential teaching to a certain extent. Combing with video and multimedia measures, teaching materials will be more intuitive than ever before, like the examples of small signal step-by-step amplifying, oscillating circuit from starting to balance, etc.

(3) Course Content Modularization. Teaching plan is always made according to textbook and the period setting. Class fragmentation research improves the in-class efficiency indeed, but not come up to expectations on information expansion. Digitization on HF Electronic Circuit with link to external open resource cuts the content into several small units in videos and 
tests so as to help students understand and assimilate. Modular process considers both the knowledge division and the micro-video design to set up 5 parts as C-Power Amplifier, LC Oscillator, AM System, FM and Demodulation System and Frequency Converter with 3 to 4 sub-modular for each.

(4) Three-dimensional Teaching Appraisal Way. It will bring big changes through flipped classroom based distributed teaching system, which asks the evaluation mode to transform from planarity to three-dimension. Such kind of examinations will not only diagnose the knowledge acquisition situation, but provide learning instructions and feedback information. In traditional appraisal system, students usually pay attentions to if there is a final review, how much is the scope, whether the problems come from postbook exercises, and so on. These, will more likely cause mind-set. Therefore, three-dimensional teaching appraisal way will evaluate from preview effect, class interaction and post-class feedback to adjust and stimulate.

HF Electronic Circuit flipped classroom will overturn the knowledge delivery and internalization according to the research. Course-oriented dimensionality mainly considers the different periods of a whole class teaching process, including pre-class, inclass and post-class, with environment creation and activity learning as the important link, as shown in Fig.1.

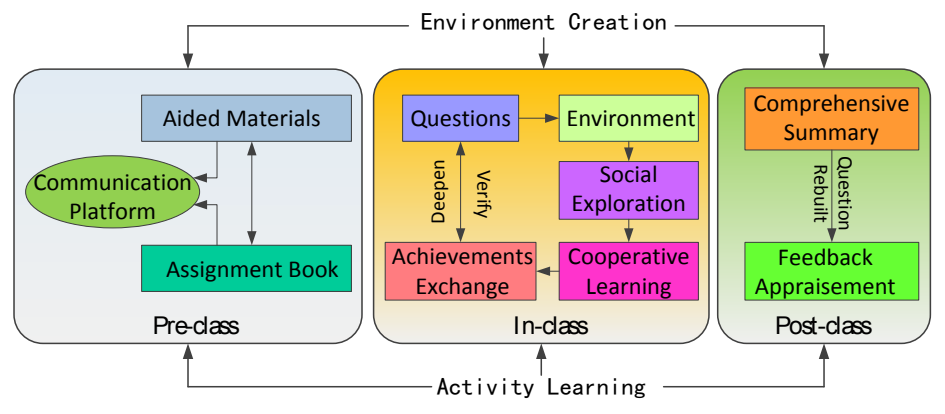

Fig. 1. Reform route map of distributed teaching based on flipped classroom

\section{A. Pre-class Learning Modular}

It relates the teaching materials preparation, including micro-video, flash, simulating figures and practical circuit, which contains three versions of primary- middle-advanced according to different course content levels and different understanding abilities. Moreover, a designed assignment book will be provided for students download, based on which they well finish the relative pre-class test and in-class question-list.

\section{B. In-class Learning Modular}

One of the most typical features of distributed teaching mechanism based on flipped classroom lies in performing the maximum pre-class learning and extending learning time on-demand. The key point is how to maximize the knowledge delivery through inclass activities. The first step is problem identification. The problems are carefully chosen and designed according to the difficult levels in reform content to guide the students thinking and exploring. The second step is cooperative learning. Four parts of LC oscillation, amplitude MDM, variode frequency modulation and frequency converter are taken as the in-class cooperative target with 3-4 discussion topics. Students will be divided into groups every 6 to 8 and evaluate with each other. The discussing results will hand in in electronic edition. Teachers may join the discussion randomly to provide direction and some solutions.

\section{Post-class Feedback Modular}

Feedback part covers evaluation but not equals to, which will perform by teachers, group members and the student himself together. This project will emphasize more on comprehensive summary including original data record, experience table, mutual evaluation and learning log. There will be a breakthrough in teaching form as well, including pre-class and in-class, network test and subjective examination, etc. to make the appraisal mechanism more comprehensive and generalized.

\section{PRACTICAL EFFeCT AND ANALYSis}

The project has carried out in 2016 HF Electronic Circuit course among three majors (communication engineering, electronic information engineering, and Electronic Information Science and Technology) of Heilongjiang University. Content modularization, flipped classroom design and distributed teaching mechanism have achieved good practical effect for students and the teaching process as well:

(1) Students could control the learning progress on-demand at dormitory, study lounge and library so as to assimilate and grasp knowledge easily and realize costumed learning to some extent. The hand-held terminal popularization and ubiquitous network access provide better flipped foundation for on-demand learning and costumed learning. The comparison of pre-class teaching 
materials visit place and media is shown in Fig.2 according to 2016 HF Electronic Circuit reform surveys, which indicates that ubiquitous and pervasive learning will greatly improve the education efficiency.

(2) Analysis on the comparison of learning materials clicks will reveal the learning situation and the difficult point, which will promote in-class teacher-student interaction. Visits of different reform content as in Fig.3 show that the visit times of $H F C$ Power Amplifier, AM System, FM System and Frequency Converter are fewer than visit times of LC Oscillatory. The difference of visit times is because LC Oscillatory is the basis of the other four systems, and the students need to review LC Oscillatory or consolidate the relative knowledge for many times when they are learning the subsequent content. Pre-class question list and assignment book enhance the in-class social contact and achievements exchange, and improve the subject consciousness in some degree.

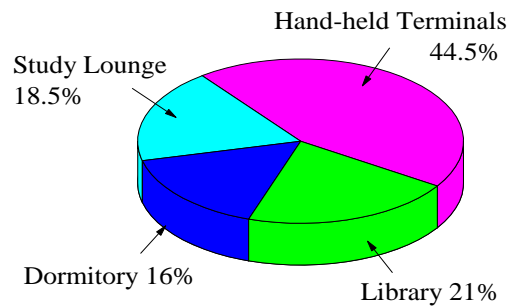

Fig.2. Sruvey of distributed learning in post-class part

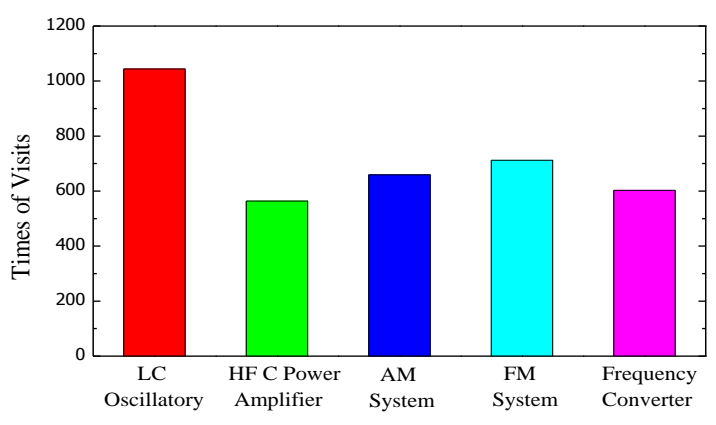

Fig.3. Comparison of visit times on distributed learning materials

(3) Sense of psychological superiority will come into being and be transformed to self-confidence. In traditional centralized teaching model, students are likely to compare with each other, especially with those who have better understanding ability. Such behavior will generate self-denial sentiment and make students start with self-categorization. Distributed teaching mechanism proposed in this paper, however, will break the centralized model to make the students control the learning schedule on-demand and help them begin to find the problem then solve the problem in some degree. Moreover, social exploration and cooperative learning in the proposed mechanism have great effect on setting up mental superiority so as to promote virtuous circle of positive study.

(4) Three-dimensional appraisal system will make the evaluation in multiple dimensions to provide a more objective result with greater guiding significance. Distributed multi-stage tests and examinations are adopted instead of the traditional complete-paper form can help students obtain adequate pre-knowledge from many tests with different forms. Cooperated in-class learning with purpose related to the reform content will re-deepen the impression, and the carefully designed tests and examinations will constantly consolidate the knowledge to promote the internalization.

Distributed teaching model based on flipped classroom for HF Electronic Circuit has obtained remarkable effect by carrying out in 3 majors of communication engineering, electronic information engineering, and Electronic Information Science and Technology with about 350 students. The teaching mechanism proposed is able to expand to other important professional courses like Communication Theory and Sensor Network etc. with the same pattern, so as to make contributions to improving the talent training quality in electronic information field and the teaching and scientific research level for the entire discipline.

\section{ACKNOWLEDGMENT}

This research was financially supported by the 2016 Higher education teaching reform project of Heilongjiang University under Grant 2016B19.

\section{REFERENCES}

[1] S. Denchev, I. Pavlova, and P. Miriyana, "The Reform of Higher Education through Alternative University Teaching Models", Creative Education, vol. 7, pp. 302-306, 2016.

[2] Q. J. Wang, J. T. Wu, "Research on University Teaching Pattern Reform based on Innovative Education Concept", International Conference on Education, Management, Computer and Society(EMCS2016), pp. 1608-1610, 2016.

[3] Q. Q. Zhong, "Educational Academic Research”, Education Reference, vol. 2, p. 5, 2016.

[4] Z. H. Wang, "Knowledge Internalization and Pattern Design based on Flipped Classroom”, Journal of Heilongjiang College of Education, vol. 35, pp. 42-44, 2016.

[5] Q. T. Wang, W. Bao, "Hybrid Teaching Research on HF Electronic Circuit based on Flipped Classroom”, Today Science and Technology, vol. 12, pp. 56-58, 2015. 